\title{
Hybrid Raman/FBG-based Sensing for Simultaneous Point Dynamic Strain and Distributed Temperature Measurement
}

\author{
Farhan Zaidi $^{1}$, Tiziano Nannipieri ${ }^{*}$, Marcelo A. Soto ${ }^{1}$, Alessandro Signorini ${ }^{1}$, \\ Gabriele Bolognini $^{1,2}$ and Fabrizio Di Pasquale ${ }^{1}$ \\ 1: Scuola Superiore Sant'Anna, via G. Moruzzi 1, 56124 Pisa, Italy. \\ 2: Consiglio Nazionale delle Ricerche, IMM Institute, Bologna, Italy \\ "E-Mail : t.nannipieri@sssup.it
}

\begin{abstract}
We propose and experimentally demonstrate an integrated hybrid optical fiber sensing technique that combines distributed Raman-based temperature measurements with a time-domain approach for FBG dynamic interrogation using a shared optical source and receiver block. OCIS codes: (060.2370) Fiber optics sensors; (190.5650) Raman effect; (060.3735) Fiber Bragg gratings
\end{abstract}

\section{Introduction}

Two distinct approaches are typically used in fiber-optic sensing of physical parameters [1]; in the first approach (distributed sensing) the measurand is continuously sensed along the optical fiber length (commonly this involves quasi-static measurements of phenomena such as Raman and Brillouin scattering), while in the second approach (discrete or point sensing), the measurand can be obtained only at specific fiber locations (typically this allows dynamic measurements and involves the use of point fiber-sensing elements, as for instance fiber Bragg gratings, FBG). Raman-based distributed temperature sensing (RDTS) is to-date the most successful and widely adopted technology for distributed sensing [2,3], while the fiber-Bragg grating is the most commonly employed device for achieving multiplexed discrete sensing; the range of their application fields spans from transportation to industrial monitoring and leakage detection in oil\&gas wells and pipelines. However, in many application fields, such as for instance in industrial plant monitoring, each sensing technique alone cannot provide all the required information (such as e.g. slow temperature profiling throughout the plant together with dynamic vibration information at specific critical points). For these applications, a sensor should be devised for (slow) distributed temperature measurements combined with discrete dynamic strain measurements; thus, providing detection of anomalous operating conditions (i.e. overheating, leakages and fire along a pipeline or within a plant) and a simultaneous dynamical monitoring of the structure conditions (i.e. vibrations in proximity of critical locations such as joints along a pipeline or a plant).

In this paper we propose a novel highly-integrated hybrid sensing system ${ }^{1}$ that effectively combines the advantages of both RDTS and FBG-based dynamic sensing and is able to perform distributed measurements up to $20 \mathrm{~km}$ as well as dynamic strain sensing with a Nyquist limit up to $2.5 \mathrm{kHz}$ rate. An integrated sensing unit based on a shared laser source and receiver block has been developed, providing a multi-functional and cost-effective solution for instance in transportation, pipelines and industrial plant monitoring applications.

\section{Theory: Hybrid Raman-FBG sensing approach}

In conventional RDTS systems, a light pulse is launched into the sensing fiber, generating spontaneous Raman scattering (SpRS) that is measured using optical time-domain reflectometry (OTDR) technique [5]. The intensity of the anti-Stokes SpRS light component strongly depends on the fiber temperature, and a normalization with a temperature-independent OTDR trace, such as Stokes SpRS or Rayleigh component, must be carried out in order to distinguish real temperature changes from local fiber loss variations [5,6]. On the other hand, FBGs are point fibersensing devices acting as band-reflect filters whose Bragg wavelength $\left(\lambda_{B}\right)$ shifts linearly with local temperature and strain changes (due to changes in the effective index and grating pitch) [3].

The hybrid approach proposed in this paper makes use of the same pulsed laser for Raman sensing and FBG interrogation. Simultaneous FBG interrogation is carried out through an OTDR approach, which is applied to multiplexed pairs of FBGs placed close to each other along the same fiber. The basic principle of the proposed technique is illustrated in Fig. 1. The light of a narrowband pulsed laser source is used to interrogate a pair of low-reflectivity Gaussian-apodized FBGs whose reflection spectra are symmetrically shifted with respect to the central wavelength of the laser source. The two FBGs are closely spaced and spatially distanced by a few meters within a packaged coiling in order for them to be subjected to the same temperature. In the FBG pair, one grating only is attached to the structure of interest

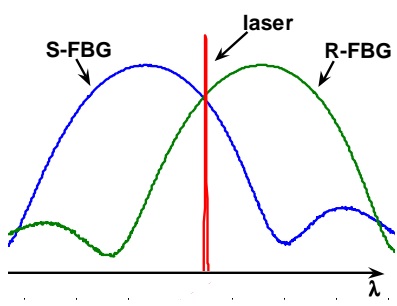

Fig. 1. Proposed dynamic strain measurement technique

\footnotetext{
${ }^{1}$ Patent pending
} 
and is then sensitive to strain (strain-sensing FBG, S-FBG), while the other one (the reference FBG, R-FBG) is loose and subjected to temperature only. In order to take into account the Gaussian shape of the FBG reflectivity, we have defined the interrogation function as the logarithm of the ratio between the integral of the FBGs reflected pulses:

$$
\rho\left(\Delta \lambda_{B}\right)=\ln \left(\int_{Z_{R-F B G}}^{Z_{R-F B G}+\Delta_{Z}} I_{R S-F B G}\left(\Delta \lambda_{B}, \xi\right) d \xi\right)-\ln \left(\int_{Z_{S-F B G}}^{Z_{S-F B G}+\Delta_{Z}} I_{R S-F B G}\left(\Delta \lambda_{B}, \xi\right) d \xi\right)
$$

where $I_{R S-F B G}\left(\Delta \lambda_{B}, \xi\right)$ is the intensity of the back-reflected pulses from FBG pair, that depends upon the Bragg wavelength shift $\Delta \lambda_{B}, Z_{R-F B G}$ and $Z_{S-F B G}$ are the longitudinal positions of FBGs along the fiber and $\Delta_{\mathrm{Z}}$ is the reflected pulsewidth. The use of a narrowband source combined with an apodized FBG pair allows us to avoid measurement distortions due to both spurious fiber loss variations and laser power fluctuations, and to obtain a monotonic (and invertible) relation between $\rho\left(\Delta \lambda_{B}\right)$ and the applied dynamic strain variations at each temperature value.

\section{Experimental Setup and Results}

The experimental setup is shown schematically in Fig. 2. The sensor reading unit (dashed line) exploits a single light source, given by a high-power rare-earth-doped fiber laser, centered at $1550.50 \mathrm{~nm}$, providing narrow-band pulses with a pulse peak power of $48 \mathrm{dBm}$, a repetition rate of $5 \mathrm{kHz}$ and $10 \mathrm{~ns}$ pulse width. A couple of variable optical attenuators (VOA) is placed to adjust the peak power level avoiding fiber nonlinearities, and an optical splitter couples the pulsed light from the sensing unit into the sensing optical fibers. The routing and filtering stage is composed of an optical circulator along the single-mode fiber (SMF) branch (employed for light coupling into the multiplexed FBG pairs and extraction of back-reflected light) and a 4-port optical filter (coupling the pulsed pump light into the multimode fiber used in distributed sensing and extracting the backscattered Stokes and anti-stokes components before the receiver stage). The integrated receiver block consists of an amplified photodiode array (a couple of avalanche photodiodes for Stokes and anti-Stokes light and a PIN diode for FBG-back-reflected light detection) and a multi-channel analog-to-digital converter (ADC) enabling simultaneous acquisition of the analog waveforms with $1 \mathrm{GS} / \mathrm{s}$ sampling frequency and subsequent processing by a PC-controlled FPGA-based board.

Since FBG-based measurements require SMFs and RDTS sensors usually employ graded-index MMF to provide acceptable performance, the output light from the sensor unit is coupled to two $20 \mathrm{~km}$ fibers (possibly within the same duplex cable), an SMF for dynamic strain sensing, and a multimode fiber (MMF) for distributed temperature sensing. Actually, the laser light (SMF output) is coupled into the filtering block and then into the MMF by a SMFMMF fiber-mode coupler to reduce higher-order mode leakage. Two FBGs with the same nominal reflectivity (5\%)

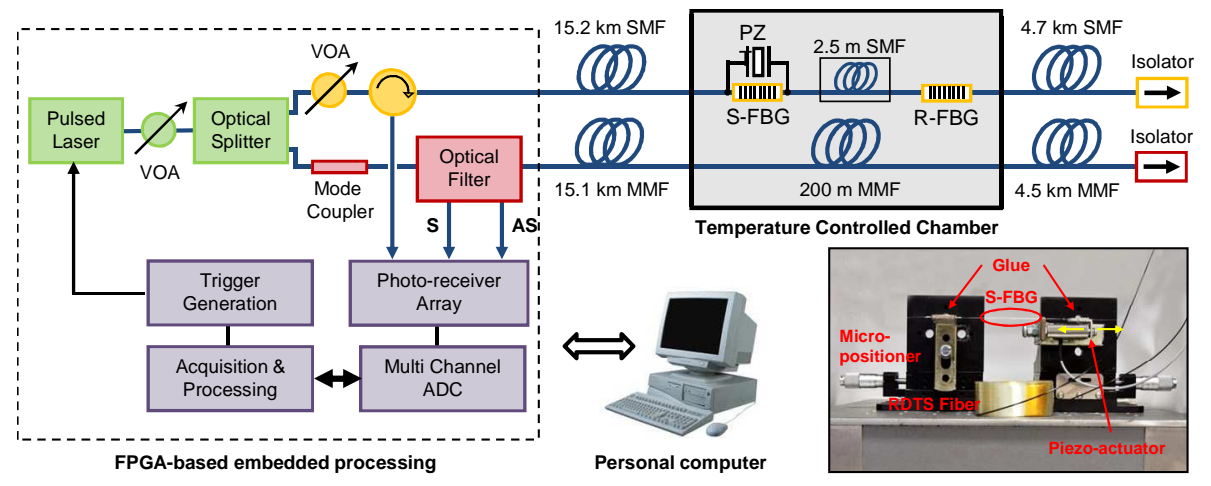

Fig 2. Experimental setup showing the sensor reading unit and the sensing fibers (Inset: picture of PZT actuation system used to apply dynamic strain to the S-FBG)

and line-width $(2.5 \mathrm{~nm})$, but centered at different wavelengths (SFBG at $1549.35 \mathrm{~nm}, \mathrm{R}-\mathrm{FBG}$ at $1551.4 \mathrm{~nm}$ ), have been separated by $2.5 \mathrm{~m}$ coiled fiber and placed at $\sim 15.2 \mathrm{~km}$ distance.

In order to analyze and validate the capability of our approach to discriminate temperature and strain, we placed $200 \mathrm{~m}$ of MMF (used in distributed temperature sensing) and both FBGs inside a temperature-controlled chamber (TCC). In addition, dynamic strain was applied to S-FBG only with a PZT-action system (Fig. 2 shows a picture with all components placed inside the TCC).

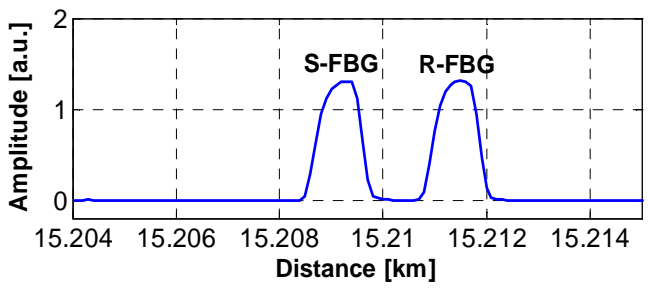

Fig. 3. FBG reflected pulses at room temperature 

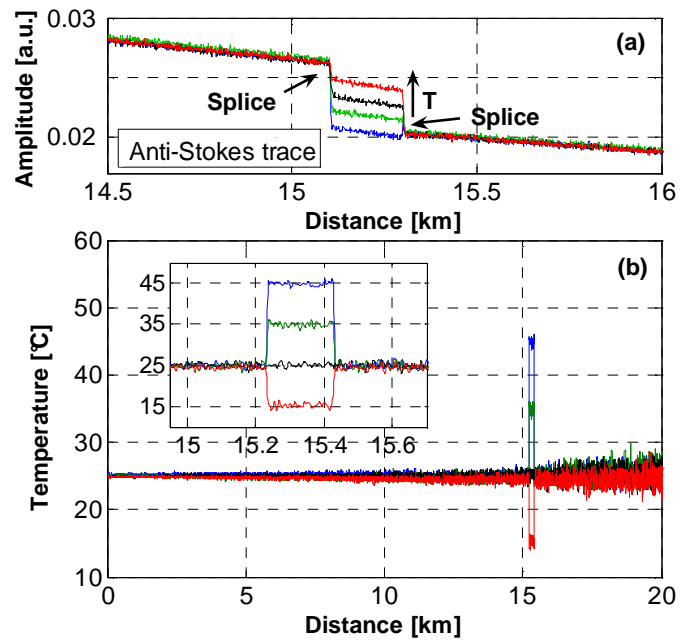

Fig. 4. (a) anti-Stokes traces at different TCC temperatures (b) Temperature profile along $20 \mathrm{~km}$ fiber

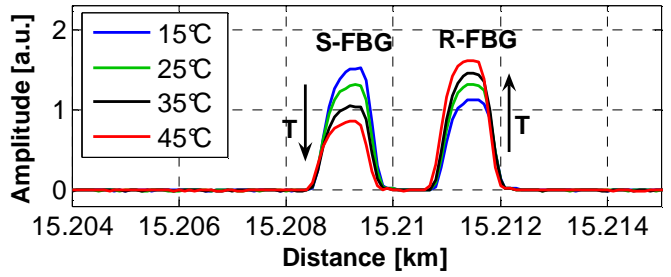

Fig. 5. FBG reflected pulses at different temperatures.
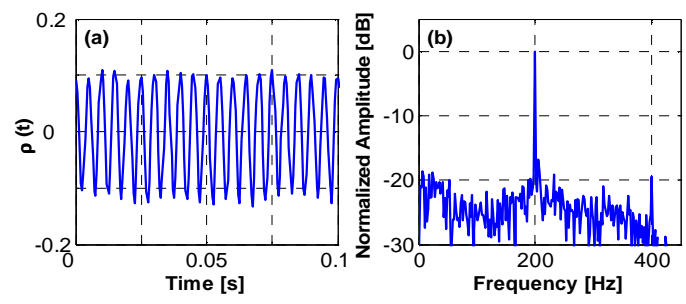

Fig. 6. Dynamic strain measurements at $200 \mathrm{~Hz}$. (a) Timedomain trace and (b) respective FFT spectrum
We applied a dynamic sinusoidal strain along longitudinal direction to the S-FBG through a piezoelectric (PZT) actuator driven by a waveform-generator $(120 \mu \varepsilon$ peak-to-peak amplitude). Figure 3 shows a single photo-detected timewaveform from the FBG pair, in which the integrals of each reflected pulse are used in Eq. (1) to infer the temperature and/or strain values. Note that temperature data from the RDTS sensing fiber has been employed to compensate the FBG straintemperature cross-sensitivity. Although the receiver bandwidth and the ADC sampling frequency would allow photo-detection up to a $200 \mathrm{MHz}$, the laser repetition rate limits the maximum FBG interrogation rate down to $2.5 \mathrm{kHz}$ (Nyquist limit).

Simultaneously to strain application, the TCC temperature was varied from $15^{\circ} \mathrm{C}$ up to $45^{\circ} \mathrm{C}$. Fig. 4(a)-(b) shows the measured intensity of anti-stokes trace and the obtained temperature profile of the $20 \mathrm{~km}$ MMF at different temperatures. A temperature resolution of $1^{\circ} \mathrm{C}$ with $1 \mathrm{~m}$ spatial resolution has been achieved at $20 \mathrm{~km}(100 \mathrm{k}$ trace averages, $30 \mathrm{~s}$ measurement time). The back reflected pulses from S-FBG and R-FBG are reported in Fig. 5 at different temperatures, clearly indicating the counteracting temperature effect for each FBG possibly affecting the strain measurements. In order to obtain temperature-independent dynamic strain measurements, suitable interrogation function values must be employed according to quasi-static temperature measurements at the FBG location obtained from the RDTS.

Finally, Fig. 6(a) shows an example of the temperatureindependent dynamic strain reconstruction (at $25^{\circ} \mathrm{C}$ ) that has been obtained using the FBGs, the interrogation function described by Eq. (1) and temperature information from the RDTS fiber; as it is evident from the figure, the final reconstructed dynamic strain trace is in a very good agreement with the known strain waveform applied to the PZT (sinusoidal, $0.2 \mathrm{kHz}$ ). Similar strain traces were obtained with all different temperatures applied to the TCC. The Fast Fourier Transform (FFT) of the measured trace is also shown in Fig. 6(b). Harmonic components are clearly visible in the amplitude spectrum, and a slight non-linear behavior of the interrogation technique can be also observed due to the presence of (significantly smaller) spurious spectral components in addition to the fundamental one. The dynamic strain resolution of our approach was estimated equal to about $20 \mathrm{n \varepsilon} /(\mathrm{Hz})^{1 / 2}$.

\section{Conclusions}

In conclusion, we have proposed and implemented a hybrid sensor scheme (integrating Raman scattering and FBGbased discrete sensing) which is able to simultaneously perform distributed temperature evaluation and dynamic discrete strain measurements using an integrated reading unit with shared source and receiver stages. Experimental results show a temperature resolution better $1^{\circ} \mathrm{C}$ with $1 \mathrm{~m}$ spatial resolution throughout $20 \mathrm{~km}$ fiber length as well as a multiplexed dynamic strain resolution of $20 \mathrm{n} /(\mathrm{Hz})^{1 / 2}$ up to $2.5 \mathrm{kHz}$ (Nyquist limit), thus providing a highperformance solution in many application domains where both dynamic and distributed measurements are required.

\section{References}

[1] F.T.S.Yu and S. Yin, Fiber Optic Sensors (M. Dekker, Inc., 2002), Chap. 4\&5.

[2] Nature Photonic, Tech. Focus 2, 143-158 (2008).

[3] K.T.V Grattan, Dr. T. Sun "Fiber optic sensor technology: an overview" Sensors and Actuators 82, 40-61 (2000).

[4] A. D. Kersey, M. A. Davis, H. J. Park, M. LeBlan, K. P. Koo “Fiber Grating Sensors” J. Lightw. Tech. 15, 1142-1463 (1997).

[5] M. A. Soto, P. K. Sahu, S.Faralli, G. Bolognini and F. Di Pasquale, "Distributed temperature sensor system based on Raman scattering using correlation code" IEEE Electronics Letter 43, 862-864 (2007).

[6] A. Signorini et al., "40 km long-range Raman based distributed temperature sensor with meter-scale spatial resolution" OFC/NFOEC (2010). 